\title{
La piel como espejo del tracto gastrointestinal
}

\section{The skin as a mirror of the gastrointestinal tract}

Martín Alonso Gómez, ${ }^{*}$ Adán Lúquez, ${ }^{2}$ Lina María Olmos. ${ }^{3}$

\footnotetext{
1 Profesor asociado de Gastroenterología, Universidad Nacional de Colombia, Unidad de Gastroenterología y Ecoendoscopia (UGEC), Hospital universitario Nacional. Bogotá D. C., Colombia.

2 Médico Internista, Gastroenterólogo, Universidad Nacional de Colombia. Bogotá D. C., Colombia.

3 Médica Dermatóloga, Universidad Militar de

Colombia, Dermatóloga del dispensario médico Gilberto Echeverry Mejía. Bogotá D. C., Colombia.

*Correspondencia: magomezz@unal.edu.co.
}

Fecha recibido: $\quad 30 / 01 / 18$

Fecha aceptado: 13/04/18

\section{Resumen pacientes y no solo centrarse en los exámenes. \\ Palabras clave \\ Piel, sangrado, endoscopia, pénfigo.}

Se presentan 4 casos de sangrado digestivo cuyas manifestaciones en la piel permitieron orientar el diagnóstico antes de la endoscopia, lo cual muestra la importancia de realizar un buen examen físico en todos los

\section{Abstract}

We present four cases of digestive bleeding whose skin manifestations guided diagnosis prior to endoscopy. These cases demonstrate the importance of a good physical examination of all patients rather than just focusing on laboratory tests.

Keywords

Skin, bleeding, endoscopy, pemphigus.
A pesar de los grandes avances tecnológicos en el diagnóstico de las enfermedades, el examen físico sigue teniendo un papel protagónico en la detección de las patologías gastrointestinales mediante un adecuado examen dermatológico. La piel es el órgano más extenso del cuerpo humano con una superficie de $2 \mathrm{~m}^{2}$, su espesor varía entre los $0,5 \mathrm{~mm}$ (en los párpados) a $4 \mathrm{~mm}$ (en el talón) y su peso aproximado es de $5 \mathrm{~kg}$ (1). Muchas manifestaciones cutáneas pueden ser indicativos de enfermedades sistémicas. Por su parte, la hemorragia de vías digestivas altas (HVDA) es la emergencia más frecuente en gastroenterología con una mortalidad entre $5 \%$ - $14 \%$; la tasa de incidencia varía geográficamente. Las principales causas son úlcera péptica (40\%) y várices esofágicas (10\%-24\%), las causas raras que corresponden a menos del $1 \%$ de las etiologías son muy difíciles de diagnosticar, pero con un buen examen físico se pueden sospechar $(2,3)$.

En este trabajo se presentan 4 causas raras de sangrado digestivo que comprometen secuencialmente el esófago, estómago, duodeno y yeyuno, cuyo diagnóstico fue orientado por las manifestaciones dermatológicas.

\section{CASO 1: PÉNFIGO VULGAR}

Paciente femenina de 46 años quien durante la hospitalización presentó un episodio de hematemesis con expulsión de membranas blanquecinas por la boca. En el examen físico presentaba múltiples erosiones y escamocostras con vesículas que cubrían toda la superficie corporal. Con diagnóstico base de pénfigo vulgar se llevó a endoscopia y se 
encontró una esfacelación del epitelio esofágico compatible con esofagitis disecante superficial (Figura 1A y B) (4).

\section{CASO 2: SÍNDROME DE RENDU-OSLER-WEBER (OWR)}

Paciente de 62 años quien ingresó a urgencias por hematemesis y melenas. En el examen físico se evidenciaron múltiples pápulas eritematovioláceas y telangiectasias en el labio, lengua y cara, con lo cual se sospechó de OWR y se llevó a endoscopia, en la que se encontraron múltiples angiodisplasias en el estómago (Figura 1C y D) (5).

\section{CASO 3: PÚRPURA DE SCHOENLEIN-HENOCH (PHS)}

Paciente de 28 años quien ingresó a urgencias por episodios de melanemesis, asociado refería artralgias, mialgias y presentaba lesiones purpúreas en las rodillas y glúteos. Con sospecha de PHS se llevó a endoscopia y se encontró en el duodeno edema grave, eritema y erosión con engrosa- miento e infiltración de la mucosa, por lo cual se tomó una biopsia (paciente con 90000 plaquetas, no presentó sangrado) con inmunohistoquímica, la cual confirmó la infiltración por inmunoglobulina A (IgA) (Figura 1E y F) (6).

\section{CASO 4: NEUROFIBROMATOSIS TIPO 1}

Paciente femenina de 29 años quien ingresó a urgencias por melanemesis, melenas y episodios recurrentes de rectorragia. En el examen físico presentó manchas "café con leche", múltiples neurofibromas en el cuerpo asociado con escoliosis, se realizó una endoscopia y colonoscopia sin observar lesiones, por un sangrado digestivo oscuro manifiesto se llevó a enteroscopia intraquirúrgica y se evidenciaron en la laparoscopia múltiples masas $(10 \mathrm{~mm}$ a $40 \mathrm{~mm}$ ) en yeyuno proximal y medio, los cuales se resecaron y el estudio histopatológico confirmó que eran neurofibromas plexiformes (no teníamos enteroscopia de uno o dos balones, por lo cual se llevó a cirugía) (Figura 1G y H) (7).

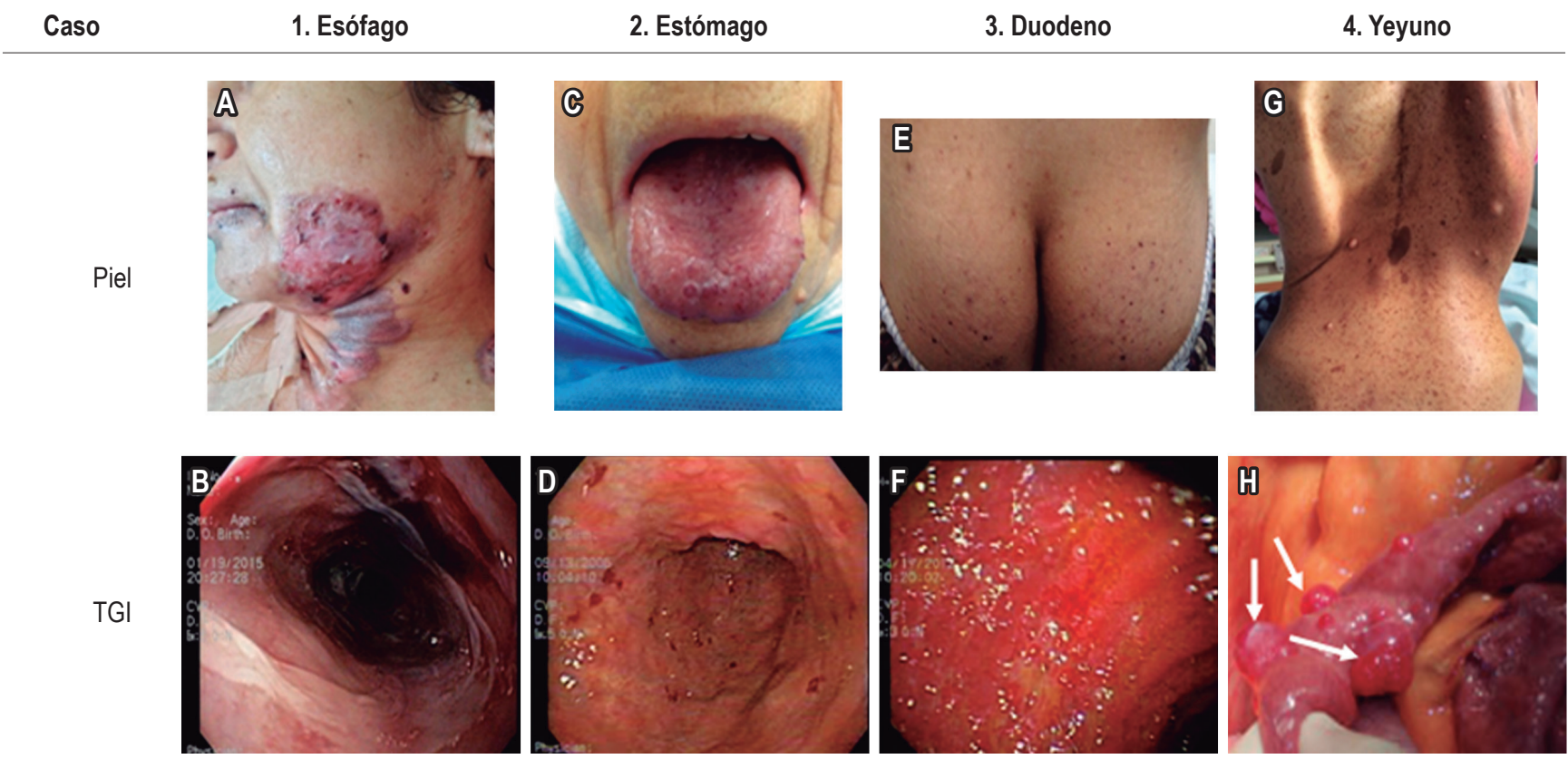

Figura 1. A. Pénfigo Vulgar. En el labio inferior se observan erosiones, costras hemorrágicas y algunas escamocostras con vesículas en la comisura labial. En la región mandibular y cuello hay erosiones, costras hemorrágicas y máculas pardovioláceas con algunas vesículas hacia la periferia de dichas lesiones. B. Esofagogastroduodenoscopia. Se observa en la parte proximal del esófago la submucosa expuesta y en la parte distal está el epitelio completamente esfacelado, por lo que se diagnostica esofagitis disecante superficial. C. Síndrome OWR. Múltiples pápulas eritematovioláceas y telangiectasias en la superficie dorsal de la lengua. D. Esofagogastroduodenoscopia. Se observan múltiples angiodisplasias en la parte distal del cuerpo gástrico, las cuales se manejaron con argón plasma. E. PHS. En los glúteos se observan pápulas purpúreas en diferentes estadios, púrpura palpable y máculas pardovioláceas postinflamatorias correspondientes a las lesiones en resolución. F. Esofagogastroduodenoscopia. Marcado edema, engrosamiento de la mucosa, eritema y erosiones en la primera porción del duodeno secundaria a la infiltración por IgA, la cual fue positiva en la inmunohistoquímica en las biopsias. G. Neurofibromatosis tipo 1. Manchas "café con leche", múltiples efélides y neurofibromas papulados y nodulares localizados en la espalda, donde también se observa escoliosis. H. Enteroscopia intraquirúrgica más laparoscopia. Se observan múltiples neurofibromas (flechas) en el yeyuno medio, los cuales fueron resecados en bloque y se realizó una anastomosis primaria. TGI: tracto gastrointestinal. 


\section{CONCLUSIÓN}

Estos casos muestran cómo, a pesar del avance en la tecnología, un buen examen físico sigue siendo fundamental en la evaluación de los pacientes ya que a través de él se puede llegar a diagnósticos insospechados cuando se va a realizar una endoscopia digestiva.

\section{REFERENCIAS}

1. Robinson ND, Hashimoto T, Amagai M, Chan LS. The new pemphigus variants. J Am Acad Dermatol. 1999;40(5 Pt 1):649-71.

2. De Peña OJ, Rodríguez O, Zambrano MT. Pénfigo vulgar oral. México D. F.: UNAM; 2000.

3. Fuentes-Guiñez P, Zambrano-Díaz MT, Rodríguez O. Características clínico-epidemiológicas de pénfigo. México D. F.: UNAM; 2000.
4. Olitsky SE. Hereditary hemorrhagic telangiectasia: diagnosis and management. Am Fam Physician. 2010;82(7):785-90.

5. Gómez M, Ruiz O. Telangiectasia hemorrágica hereditaria. Reporte de Caso. Rev Col Gastroenterol. 2015;30(4):46973. https://doi.org/10.22516/25007440.11.

6. González-Gay MA, López-Mejías R, Pina T, Blanco R, Castañeda S. IgA Vasculitis: Genetics and Clinical and Therapeutic Management. Curr Rheumatol Rep. 2018;20(5):24.

7. Hernández-Martín A, Duat-Rodríguez A. An Update on Neurofibromatosis Type 1: Not Just Café-au-Lait Spots, Freckling, and Neurofibromas. An Update. Part I. Dermatological Clinical Criteria Diagnostic of the Disease. Actas Dermosifiliogr. 2016;107(6):454-64. https://doi. org/10.1016/j.ad.2016.01.004. 